Dear Author,

Please, note that changes made to the HTML content will be added to the article before publication, but are not reflected in this PDF.

Note also that this file should not be used for submitting corrections. 


\title{
Q1 Synthesis of nitrogen-doped epitaxial graphene via plasma-assisted method: Role of the graphene-substrate interaction
}

\author{
Q2 Fabrizio Orlando ${ }^{\mathrm{a}, 1}$, Paolo Lacovig ${ }^{\mathrm{b}}$, Matteo Dalmiglio ${ }^{\mathrm{b}}$, Alessandro Baraldi ${ }^{\mathrm{a}, \mathrm{b}, \mathrm{c}}$, \\ Rosanna Larciprete ${ }^{\mathrm{d}}$, Silvano Lizzit ${ }^{\mathrm{b}, *}$ \\ a Physics Department, University of Trieste, Via Valerio 2, 34127 Trieste, Italy \\ b Elettra-Sincrotrone Trieste S.C.p.A., AREA Science Park, S.S. 14 km 163.5, 34149 Trieste, Italy \\ c IOM-CNR, Laboratorio TASC, AREA Science Park, S.S. 14 km 163.5, 34149, Trieste, Italy \\ d CNR-Institute for Complex Systems, Via Fosso del Cavaliere 100, 00133 Roma, Italy
}

\section{A R T I C L E I N F O}

Available online $\mathrm{xxxx}$

\section{Keywords:}

Graphene

Functionalization

Nitrogen doping

XPS

XPD

Iridium

\begin{abstract}
A B S T R A C T
Functionalization of graphene by substitution of the carbon with nitrogen atoms is a promising way to tailor its 19 electronic properties, but a good control over the heteroatomic configuration in the graphene network is most 20 often a difficult task. In this paper, the synthesis of N-doped graphene by nitrogen plasma treatment of 21 graphene/Ir(111) is presented. The formation of substitutional, pyrrolic and pyridinic nitrogen is analyzed by 22 means of X-ray photoelectron spectroscopy (XPS) and X-ray photoelectron diffraction (XPD). The graphene-Ir 23 interaction is suggested to control the variation in the relative concentration of the nitrogen species. Annealing 24 of the sample also leads to modifications of the nitrogen species incorporated in the graphene layer. Furthermore, 25 the connection of the substitutional nitrogen arrangement with its corresponding spectroscopic fingerprint is 26 unequivocally confirmed by XPD measurements which give also a direct insight on the local geometry of the 27 nitrogen atoms incorporated in the carbon network.
\end{abstract}

(C) 2015 Published by Elsevier B.V.

\section{Introduction}

The combination of many outstanding properties in a single material makes graphene (GR) really attractive for a number of technological applications. In this regard, the use of graphene single layers into highperformance integrated circuits is one of the most intriguing prospects. However, the absence of a gap in its band structure, sets severe limits on the use of graphene in, e.g., transistors because of the low on-off ratio achievable [1]. This is the reason why the opening and fine tuning of an energy gap in the band structure of graphene has been an issue of fundamental importance since its discovery. Different routes have been followed to tailor the electronic properties of graphene. The first to be explored were methods based on the morphology control over the graphene structure. For instance, by making graphene nanoribbons it is possible to laterally confine the charge carriers. In nanoribbons, different types of edges show either metallic (zigzag edge) or semiconducting (armchair edge) character [2]. Furthermore, the possibility of opening a band gap in bilayer graphene by applying a strong electric field [3] or by selectively controlling the carrier density in each layer

\footnotetext{
* Corresponding author

E-mail address: silvano.lizzit@elettra.eu (S. Lizzit).

1 Present address: Laboratory of Radiochemistry and Environmental Chemistry, Paul Scherrer Institut, 5232 Villigen, Switzerland.
}

[4] was also explored. However, these approaches resulted in a small 52 band gap or in a strong degradation of the carrier mobility [1]. 53

A promising alternative approach to modify the electronic proper- 54 ties of graphene is by chemical doping, which can be achieved by 55 patterning the graphene surface with atoms [5] or molecules [6], or by 56 introducing heteroatoms in the carbon network [7]. In the latter case, 57 the substitution of carbon with nitrogen or boron atoms is of consider- 58 able interest not only because the shift of the energy of the Dirac point 59 leading to $\mathrm{n}$ - or $\mathrm{p}$-doped graphene could be achieved, but also because 60 the characteristic features of the graphene electronic band structure 61 could be modified [8]. For $\mathrm{sp}^{2}$ carbon structures, like graphene or carbon 62 nanotubes, the incorporation of nitrogen in the lattice has been already 63 demonstrated to be a robust and efficient method to tune the electronic 64 properties with only minor structural perturbations, due to the possibil- 65 ity to form covalent bonds $[9,10]$.

Several methods have been developed to synthesize N-doped 67 graphene. For instance, direct synthesis of N-doped graphene can be 68 achieved by chemical vapor deposition (CVD) [11] or arc discharge 69 [12] approaches. On the other hand, post-synthesis methods are also 70 widely used, such as thermal annealing [13] in ammonia $\left(\mathrm{NH}_{3}\right)$ atmo- 71 sphere or plasma treatment $[14,15]$. Although different atomic arrange- 72 ments of the nitrogen atoms in the carbon network are possible (some 73 of them are displayed in the top panel of Fig. 1), three main configura- 74 tions have been characterized namely graphitic, also known as substitu- 75 tional, pyridinic and pyrrolic nitrogen. Several characterization tools 76 


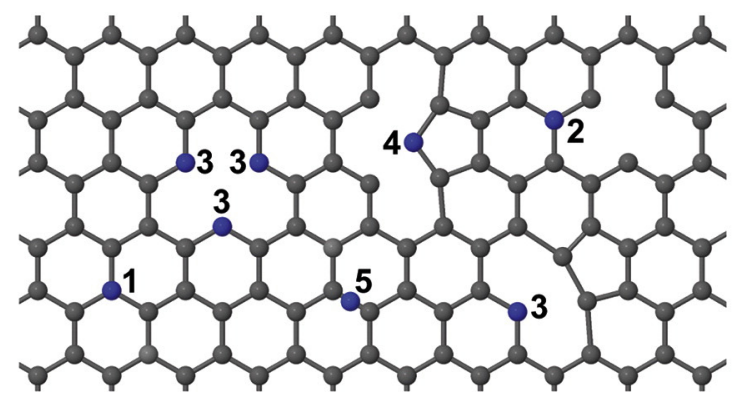

\section{Graphitic1 \\ 2 Graphitic2 \\ 3 Pyridinic \\ 4 Pyrrolic}

5 Interstitial / Adatom
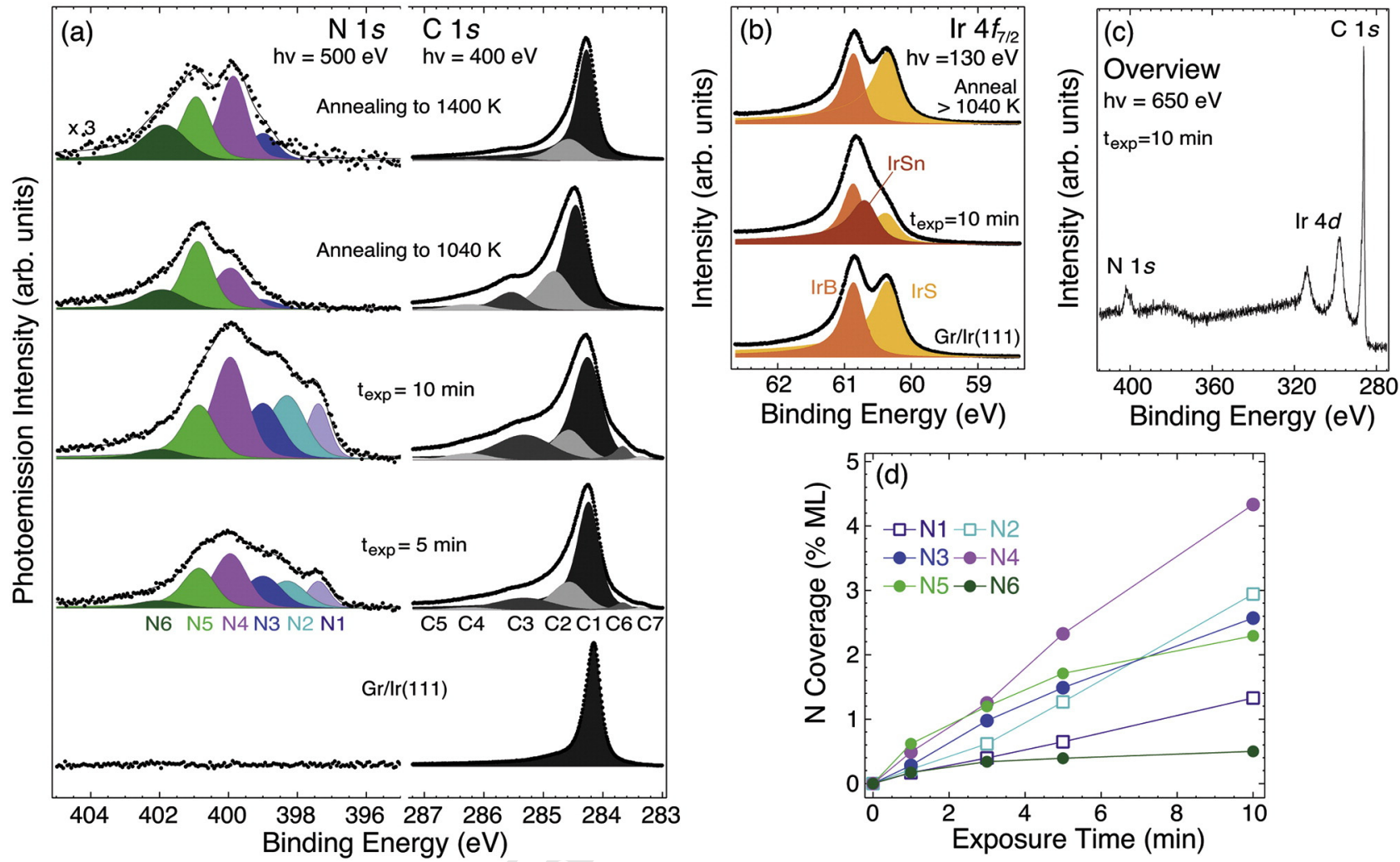

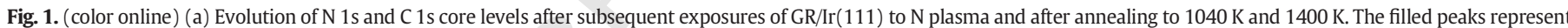

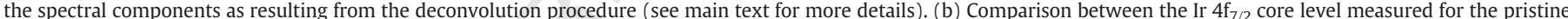

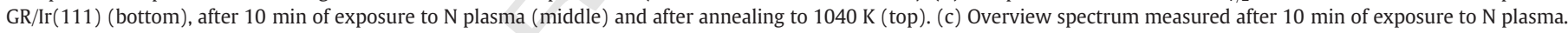

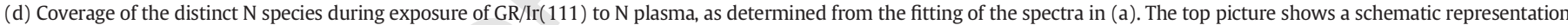

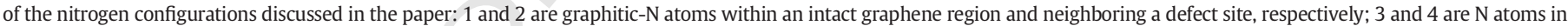
pyridinic and pyrrolic configuration while 5 is $\mathrm{N}$ atoms adsorbed on the graphene basal plane or in interstitial position.

based on microscopy and spectroscopy have been used to investigate the $\mathrm{N}$-doped graphene layers (e.g. scanning tunneling microscopy (STM) [16,17], transmission electron microscopy (TEM) [18], X-ray photoelectron spectroscopy (XPS) $[9,10]$ and Raman spectroscopy $[11,19])$, but the connection between the nitrogen bonding configuration and the synthesis method is not always straightforward.

Motivated by this lack of information, we exploited the potential offered by XPS and X-ray photoelectron diffraction (XPD) techniques to study the functionalization and to determine the surface structure of a N-doped graphene layer produced upon nitrogen plasma exposure of the GR/Ir(111) interface. In this paper we illustrate how a combined XPS and XPD investigation can provide insight into the correlation between the electronic and structural properties of $\mathrm{N}$-doped graphene.

\section{Material and methods}

The experiments were performed at the SuperESCA beamline of Elettra, the synchrotron radiation facility in Trieste, Italy. The $\operatorname{Ir}(111)$ single crystal was cleaned by repeated cycles of $\mathrm{Ar}^{+}$sputtering and annealing in $\mathrm{O}_{2}$ atmosphere between 600 and $1100 \mathrm{~K}$, followed by hydrogen exposure at $800 \mathrm{~K}$ to remove the residual oxygen. The 95 graphene monolayer was grown on $\operatorname{Ir}(111)$ by doing 10 cycles of 96 temperature-programmed growth, consisting in dosing ethylene at 97 $520 \mathrm{~K}$ and annealing to $1470 \mathrm{~K}$, followed by a prolonged annealing at 98 high temperature with a base ethylene pressure of $1 \times 10^{-7}$ mbar. 99 This procedure ensures the growth of a complete layer of graphene 100 that does not leave bare Ir regions. The as-grown graphene layer was 101 then exposed to nitrogen plasma at room temperature using a Gen2 102 plasma source from Tectra $\mathrm{GmbH}$, configured as atom source without 103 ion-trap. Within this mode of operation the beam is mainly composed 104 by neutral atoms with a fraction of residual ions with energy of about 105 $25 \mathrm{eV}$, defined by the intrinsic plasma potential. Different conditions 106 such as $\mathrm{N}_{2}$ pressure, position of the sample surface with respect to 107 the source and exposure time were tested. An efficient nitruration 108 of graphene was achieved for exposures with $\mathrm{N}_{2}$ base pressure of 109 $2.5 \times 10^{-5}$ mbar performed with the Ir surface placed in front of the 110 plasma source.

The high-energy resolution XPS spectra of the $\operatorname{Ir}_{4} 4 \mathrm{f}_{7 / 2}, \mathrm{C} 1 \mathrm{~s}$ and $\mathrm{N} 1 \mathrm{~s} 112$ core levels were measured in normal emission (in the present setup this 113 corresponds to $70^{\circ}$ of incidence of the photon beam) using photon 114 
energies of 130, 400 and $500 \mathrm{eV}$, respectively, with an overall energy resolution ranging from 40 to $100 \mathrm{meV}$. The core level binding energy scale was referred to the Fermi level of the Ir substrate measured in the same conditions. The spectra were fitted with Doniach-Šunjić functions [20] convoluted with a Gaussian, and a linear background. The nitrogen concentration was determined from survey spectra measured at $650 \mathrm{eV}$ by calculating the ratio of the $\mathrm{N} 1 \mathrm{~s}$ and $\mathrm{C} 1 \mathrm{~s}$ intensities properly normalized for the respective photoemission cross section at this photon energy.

The XPD measurements of the N1s core level were performed with photon energy of $500 \mathrm{eV}$ corresponding to an electron kinetic energy of $100 \mathrm{eV}$. The diffraction patterns were measured over an azimuthal sector of $120^{\circ}$, from normal $\left(\theta=0^{\circ}\right)$ to grazing emission $\left(\theta=80^{\circ}\right)$. The modulation functions were obtained for each polar emission angle $\theta$ from the peak intensity $I(\theta, \Phi)$ as $\left(I(\theta, \Phi)-I_{0}(\theta)\right) / I_{0}(\theta)$, where $I_{0}(\theta)$ is the average value of each azimuthal scan. The simulations were performed with the Electron Diffraction in Atomic Cluster (EDAC) package [21] for a free-standing, flat graphene layer with a $\mathrm{N}$ atom in graphitic configuration as emitter. The inclusion of the Ir substrate in the simulations, does not produce appreciable differences in the XPD patterns, because of the lattice mismatch between graphene and $\operatorname{Ir}(111)$. Indeed, any $\mathrm{N}$ atom in graphitic configuration within a moiré unit cell does not have a specific local geometry with respect to the Ir atoms thus smearing out the diffraction from the Ir substrate.

\section{Results and discussion}

As a first step, we followed the evolution of the N 1s, C 1 s and $\operatorname{Ir} 4 f_{7 / 2}$ core level spectra as a function of the nitrogen plasma exposure on $\mathrm{GR} / \mathrm{Ir}(111)$. We performed 5 cycles for a total dosing time of $10 \mathrm{~min}$, as shown in Fig. 1. The final nitrogen concentration was estimated to be $0.14 \mathrm{ML}$ with respect to graphene, as determined from the overview spectrum of Fig. 1c. From the beginning of nitrogen exposure (Fig. 1a), the $\mathrm{N} 1 \mathrm{~s}$ core level region exhibits a broad spectrum, where six different components have to be set in order to fit the uptake series. These components arise from nitrogen atoms in different chemical environments: N1 (397.4 eV), N2 (398.3 eV), N3 (399.0 eV), N4 (400.0 eV), N5 $(400.9 \mathrm{eV})$ and N6 (401.9 eV). Fig. 1d shows the evolution of the N 1s components as a function of dosing time. It appears quite clear that N5 and N6 display the same behavior approaching almost saturation after a dosing time of $10 \mathrm{~min}$, while the other components keep on increasing almost linearly. The N2-N6 components fall in the binding energy range of the graphitic (N5, N6), pyrrolic (N4) and pyridinic (N2, N3) nitrogen, as previously reported [9,11,13,15,19,22-26], but their assignment to specific nitrogen species is not straightforward. Indeed, other configurations may be possible, including nitrogen adatoms or interstitial nitrogen as most of the nitrogen plasma impinging on the surface is composed by $\mathrm{N}$ atoms that could adsorb on GR. The Ir substrate may also play an important role in GR functionalization [5,27], even if GR/Ir(111) is a weakly interacting system [28,29]. Based on the XPD investigation described below and according to Refs. [15] and [30], we attribute N5 and N6 to graphitic-like N atoms, placed within an intact graphene region and neighboring a defect site, respectively (denoted as graphitic1 and graphitic2 in the top part of Fig. 1). The position of $\mathrm{N} 1$ is in the range of the binding energies measured for nitrogen atoms adsorbed on the $\operatorname{Ir}(111)$ surface dosed with $\mathrm{NH}_{3}$ [31]. Also in the present study we observed a single $\mathrm{N} 1 \mathrm{~s}$ peak centered at $397.4 \mathrm{eV}$ after nitrogen plasma dose on the clean $\operatorname{Ir}(111)$ surface (top curve of Fig. 2c). Therefore, we assign N1 to nitrogen atoms that intercalate below graphene and chemisorb on the Ir substrate. As illustrated in Fig. 1a, the relatively high intensity of $\mathrm{N} 1$ indicates that a sizeable amount of $\mathrm{N}$ atoms intercalate underneath the graphene layer. The intercalation process may take place at pre-existing graphene defects or wrinkles, as already suggested for $\mathrm{O}_{2}$ intercalation on $\mathrm{GR} / \mathrm{Ir}(111)$ [32]. However, in the present case it is more likely that the nitrogen ions from the plasma source introduce defects in graphene: the carbon network is damaged upon nitrogen plasma exposure, thus fostering further 179 intercalation.

The growth of the different components in the $\mathrm{N} 1 \mathrm{~s}$ core level region 181 is paralleled by a broadening of the $C 1$ s spectrum (Fig. 1a) reflecting the 182 changes of the graphene lattice induced by the nitrogen plasma treat- 183 ment which eventually causes also the incorporation of nitrogen 184 atoms in the graphene network. As a result, the main C 1s peak (C1) re- 185 lated to $\mathrm{sp}^{2}$ bonded C-C atoms broadens and shifts towards higher bind- 186 ing energy with respect to the value measured for the clean graphene 187 (284.14 eV), and several other components appear at higher and 188 lower binding energy: C2 (284.54 eV), C3 (285.26 eV), C4 (286.20 eV), 189 C5 $(287.10 \mathrm{eV}), \mathrm{C} 6(283.65 \mathrm{eV})$ and C7 $(283.35 \mathrm{eV})$. It has to be noted 190 that the total intensity in the $C 1 \mathrm{~s}$ region keeps constant during 191 nitruration. While C6 and C7 could be attributed to carbon vacancies 192 [33] and to $C$ atoms at the Ir steps [28], respectively, the interpretation 193 of the C2-C5 components is not straightforward. Indeed, their large 194 width indicates that they can include contributions from different con- 195 figurations of the carbon atoms. We tentatively assign C3-C5 peaks to 196 $\mathrm{sp}^{2}$ and $\mathrm{sp}^{3} \mathrm{C}-\mathrm{N}$ bonds [34] while C2 may be attributed to the carbon 197 atoms second-nearest neighbors to a graphitic-N, similarly to the case 198 of a planar 3-fold C-O configuration resulting from the inclusion of an 199 oxygen atom in a carbon vacancy [33]. However, calculations similar 200 to those performed in Ref. [33] would be required in order to clarify 201 this issue.

The variations in the chemical composition of the graphene layer 203 lead to noticeable changes also in the Ir $4 \mathrm{f}_{7 / 2}$ spectra (Fig. 1b). The Ir 204 $4 f_{7 / 2}$ spectrum of pristine GR/Ir(111) consists of two components, 205 representing bulk (IrB at $60.84 \mathrm{eV}$ ) and surface (IrS at $60.31 \mathrm{eV}$ ) 206 atoms, and it is comparable to that of clean $\operatorname{Ir}(111)$ [35] because of the 207 low interaction between the graphene layer and the substrate [28]. 208 Upon nitrogen plasma treatment, a new component IrSn appears 209 between the surface and the bulk peaks at $60.70 \mathrm{eV}$, which grows at 210 the expense of the surface component IrS, due to the adsorption of the 211 intercalated nitrogen and to the interaction of the N-doped graphene 212 with the iridium substrate.

With the aim of determining the role of the Ir substrate in nitrogen 214 functionalization of $\mathrm{GR} / \operatorname{Ir}(111)$, we performed identical nitrogen 215 plasma treatments on a quasi free-standing graphene layer obtained 216 by intercalating oxygen under graphene [32]. Fig. 2 shows the N 1s 217 core levels of the GR/O/Ir(111) system during nitrogen plasma treat- 218 ment. The comparison between the spectra measured after 10 min 219 plasma exposure on GR/Ir(111) and GR/O/Ir(111) shows that the over- 220 all $\mathrm{N}$ coverage decreases to $0.1 \mathrm{ML}$ in the latter case. On the other hand 221 big differences in the relative concentration of the nitrogen species are 222 quite evident from the comparison of Fig. 2b with Fig. 1d. More precise- 223 ly, the N1 intensity, i.e. the amount of nitrogen adsorbed on the Ir 224 substrate, is practically unchanged, N5 and N6 increase, N2 slightly 225 decreases while N3 and N4 drop to half of their respective intensity for 226 the $\mathrm{Gr} / \mathrm{Ir}(111)$ system. As a result, the absolute coverage of graphitic-N 227 species (N5 + N6) goes from 0.028 ML to 0.036 ML and their concentra- 228 tion with respect to the other nitrogen species bound to graphene 229 $(\mathrm{N} 2+\mathrm{N} 3+\mathrm{N} 4)$ increases from $28 \%$ to $60 \%$.

The comparison between the two experiments described above 231 clearly highlights the influence of the substrate on the functionalization 232 of the graphene layer. The GR-Ir interaction, albeit weak, fosters the for- 233 mation of the pyridinic and pyrrolic nitrogen species. In addition, the 234 formation of graphitic-N can be hindered in the valleys of the moiré cor- 235 rugation of GR/Ir(111) [28], where the bonding between C and Ir atoms 236 is stronger. It is worth noting that a number of studies have previously 237 demonstrated the crucial role of the weak interaction of graphene 238 with the underlying $\operatorname{Ir}(111)$ substrate and, in particular, of the template 239 structure of this interface. For instance, the coupling to the substrate 240 controls the patterned adsorption of hydrogen on GR/Ir(111) [5,27], 241 drives the (de)oxidation processes during thermal reduction of 242 graphene oxide [36], allows to fine tune the morphology of nanoclusters 243 $[37,38]$ an can even affect charge transfer processes of photo-excited 244 

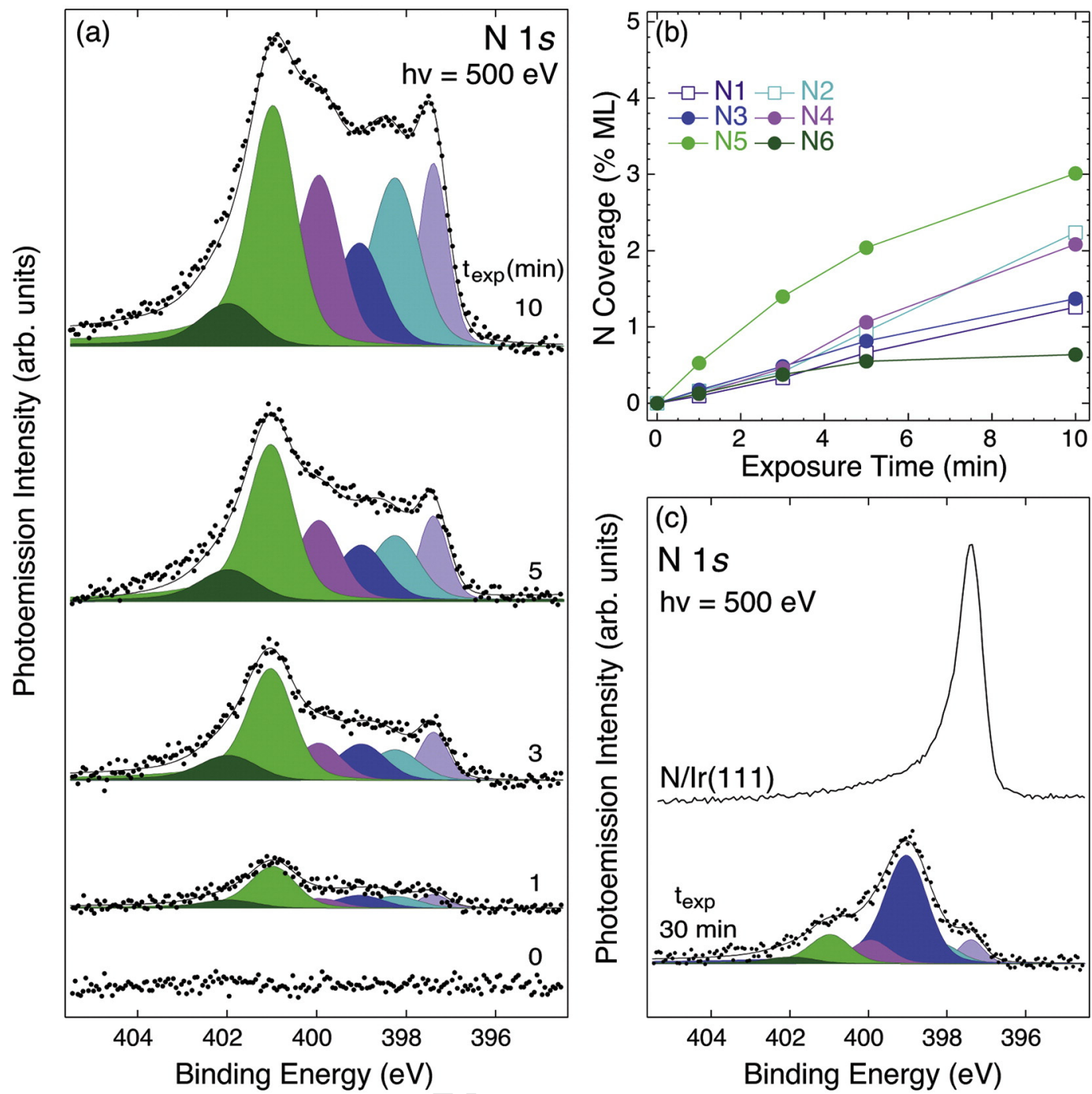

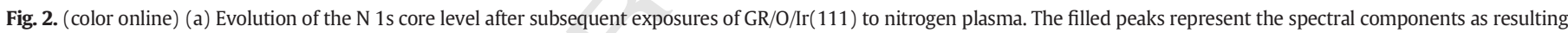

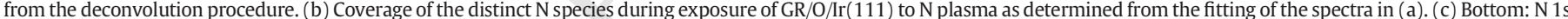

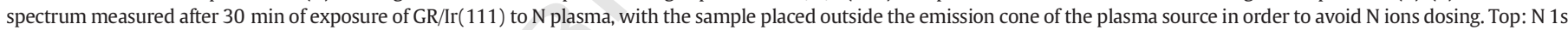
spectrum measured after saturation of the bare $\operatorname{Ir}(111)$ surface with atomic $\mathrm{N}$ from the plasma source.

electrons [39]. On the other hand, the suppression of the GR-Ir interaction upon oxygen intercalation leads to significant reduction of the pyridinic and pyrrolic-N content, suggesting that these configurations are stabilized by the presence of the Ir surface.

In order to highlight the contribution of the $\mathrm{N}$ atoms we dosed for 30 min the sample rotated with respect to the emission cone of the source in order to avoid hitting the surface with nitrogen ions. The bottom spectrum in Fig. 2c shows the resulting N1s spectrum. As the carbon network should be less damaged by this treatment with respect to the standard exposure geometry (Figs. 1 and 2), it is reasonable to assume that most of the nitrogen atoms are now adsorbed on graphene or in interstitial positions and do not form pyridinic species which need the formation of a defect. Indeed, the resulting N1s spectrum is composed mainly by one peak, which coincides with N3. This gives a strong indication that the adatoms contribute to the N3 component. Moreover, this finding suggests that in order to form graphitic, pyridinic and pyrrolic species, energetic nitrogen ions are required to break the $\mathrm{C}-\mathrm{C}$ bonds. The chemical doping of graphene is therefore achieved only if defects are introduced in the carbon network by kinetic impact with the energetic ions.

In order to shed light on the thermal stability of the N-doped graphene we performed a thermal desorption experiment while monitoring the N 1s core level region. The central panel in Fig. 3a 267 shows the intensity plot derived from the Temperature Programmed 268 XPS (TP-XPS) [40] N 1s spectra acquired while annealing the sample up 269 to $1040 \mathrm{~K}$. The $\mathrm{N} 1 \mathrm{~s}$ spectra measured at room temperature before and 270 after the annealing are reported at the bottom and top of Fig. 3a, respec- 271 tively, while the quantitative analysis of the TP-XPS spectra is displayed in 272 Fig. 3b. In Fig. 1a the corresponding C 1s spectra are shown. Upon anneal- 273 ing, the total amount of nitrogen goes from $0.14 \mathrm{ML}$ to $0.06 \mathrm{ML}$, the rela- 274 tive concentration of the nitrogen species changes and the $C$ is peak 275 narrows losing about $5 \%$ of its initial intensity. The most interesting aspect 276 of the annealing process is the conversion of part of the nitrogen species 277 into graphitic- $\mathrm{N}$ between 400 and $900 \mathrm{~K}$, as indicated by the significant 278 increase of N5 and N6 at the expense of all other components in this tem- 279 perature range. It should be noted that the rearrangement of nitrogen 280 species upon annealing has been already observed for similar interfaces 281 $[9,23,41]$. The behavior of the atomic nitrogen component N1, which 282 disappears above $800 \mathrm{~K}$, resembles that observed on the pristine 283 $\operatorname{Ir}(111)$ surface, where the nitrogen atoms desorb as $N_{2}$ between 400284 and $700 \mathrm{~K}$ [31]. The intensity of the N2, N3 and N4 components decreases 285 with temperature: above $800 \mathrm{~K} \mathrm{~N} 2$ is completely suppressed, N3 reaches 286 an almost constant value that is about a third of its initial value while N4 287 decreases almost linearly in the whole temperature range. 

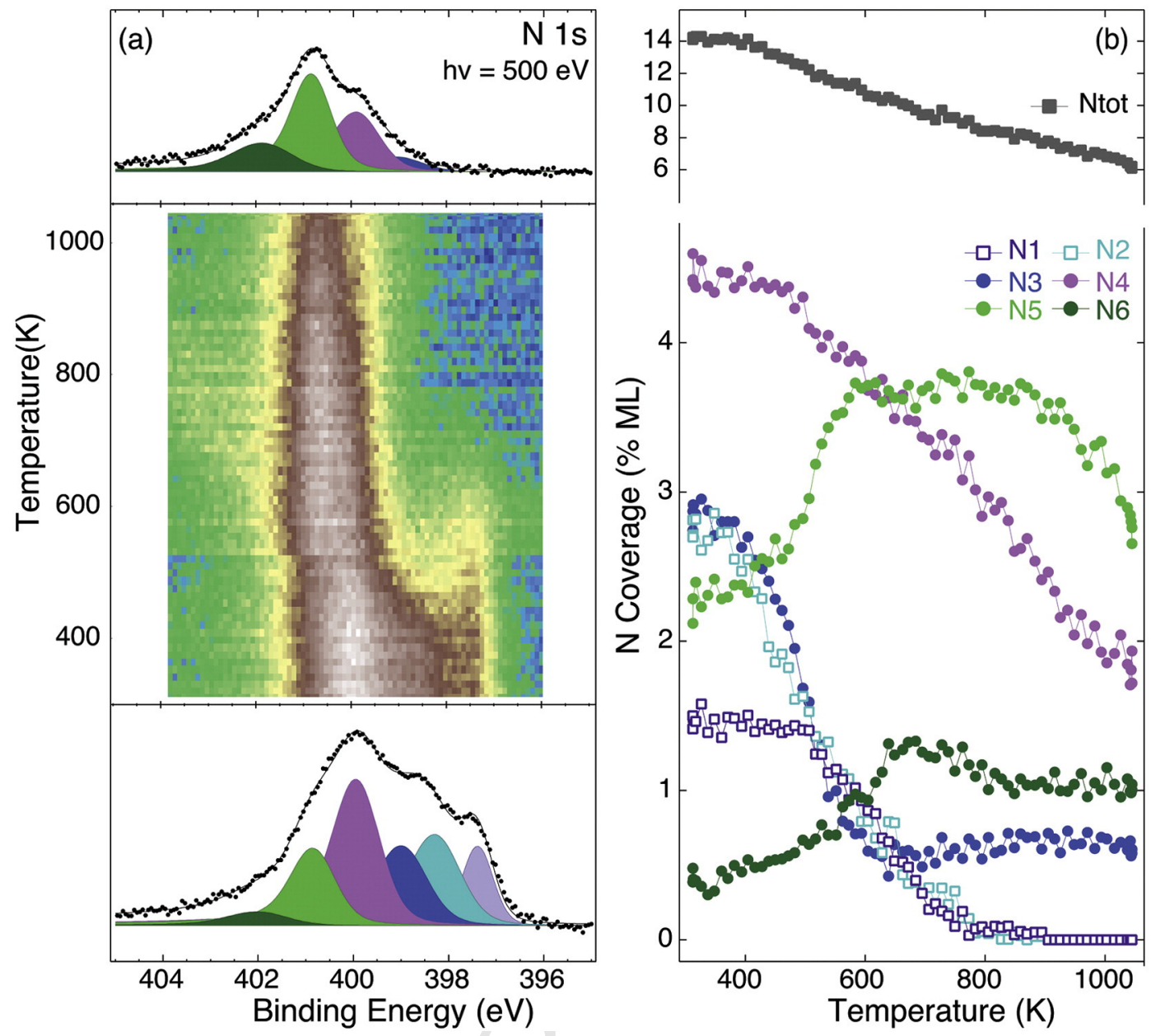

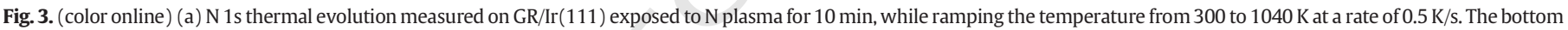

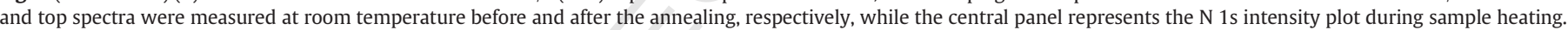

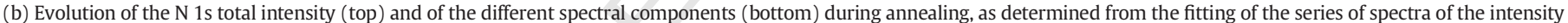
plot of the central panel in (a).

As graphitic- and pyridinic-N are expected to be the most stable species at high temperature [15,42], a large fraction of N3 has to be assigned to nitrogen atoms in pyridinic configuration. The correlation between the intensity of graphitic and pyridinic components in Fig. 3b suggests that the main conversion process is from the pyridinic to the graphitic configuration. This behavior might be interpreted in terms of a thermally activated mobility of the vacancies in the graphene network facilitating their diffusion far away from the pyridinic-N configuration, which therefore converts into graphitic-N. It has been previously reported that the $\mathrm{N} 1 \mathrm{~s}$ peak of pyridinic-N on $\mathrm{GR} / \mathrm{Ni}(111)$ shifts to smaller binding energy after gold intercalation, because of the suppressed substrate interaction of pyridinic-N [25]. In our case, two pyridinic species can coexist as the result of a relatively strong (weak) interaction of the Ir surface at the valley (hill) regions of the GR/Ir(111) moire structure. The conversion of pyridinic- to graphitic$\mathrm{N}$ could then take place only on the hills of the moiré, where the defect diffusion is larger [43]. Given this consideration we hypothesize that N2 and N3 are associated to pyridinic nitrogen atoms in the valleys and hills of the moiré superstructure, respectively, with N3 containing a contribution also from interstitial/adatom $\mathrm{N}$ (see above). This is in line with the observed residual intensity of $\mathrm{N} 3$, i.e. the strongly interacting pyridinic component, and quenching of N2, the weakly interacting component, after sample annealing as illustrated in Fig. 3b. The interaction with the substrate can even prevent the conversion of part of the pyrrolic into substitutional $\mathrm{N}$, thus justifying the high intensity of the $\mathrm{N} 4$ component after annealing, especially when compared to the pyridinic part. Regarding this point, however, one has also to take into account 315 their different concentrations before the thermal treatment, with the 316 intensity of the pyrrolic nitrogen component being almost two times 317 larger than the pyridinic-N species (Fig. 3b).

After the annealing to $1040 \mathrm{~K}$ the sample contains nitrogen atoms 319 mostly in graphitic configuration with a coverage of $0.04 \mathrm{ML}$. Further 320 annealing to $1400 \mathrm{~K}$ leads to a reduction of the graphitic part to 321 0.014 ML (top spectrum of Fig. 1a). Remarkably, in both cases the Ir 322 $4 \mathrm{f}_{7 / 2}$ spectrum (Fig. 1b, top) is almost indistinguishable from that of 323 the pristine GR/Ir(111) (Fig. 1b, bottom). Indeed, upon annealing the 324 IrSn component is completely depleted, while the IrS intensity is fully 325 restored. This suggests that the interaction between the graphene 326 layer doped mainly with graphitic-N and the Ir surface is weak and re- 327 sembles that of the as-grown graphene on $\operatorname{Ir}(111)$. This result indicates 328 that the graphene layer is not significantly deformed upon inclusion of 329 substitutional nitrogen.

330

Fig. 4 shows the binding energy shift of the main graphene peak 331 $\mathrm{C} 1$ as a function of the coverage of graphitic and pyridinic-N 332 $(\mathrm{N} 2+\mathrm{N} 3+\mathrm{N} 5+\mathrm{N} 6)$ during the nitrogen plasma exposure (blue 333 markers) and after annealing to high temperature (red markers) 334 together with the $\mathrm{N}$ coverage behavior upon annealing shown in 335 the inset. Upon plasma exposure the nitrogen doping of graphene in- 336 duces a progressive shift of $\mathrm{C} 1$ towards higher binding energy up to 337 $\sim 100 \mathrm{meV}$. After annealing to $1040 \mathrm{~K}$, when the largest fraction of the 338 nitrogen atoms is in graphitic configuration, the shift of C1 further 339 increases by $200 \mathrm{meV}$. The additional annealing to $1400 \mathrm{~K}$ induces a 340 


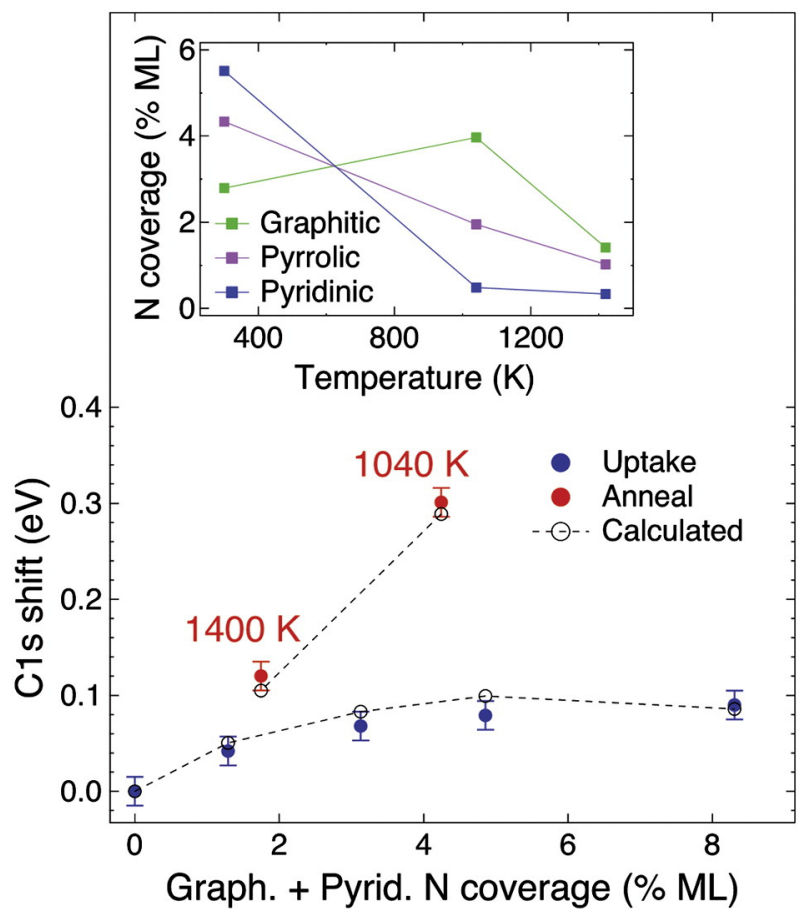

Fig. 4. (color online) Binding energy shift of the $\mathrm{sp}^{2}$ component ( $\mathrm{C} 1$ in Fig. 1 ) in the $\mathrm{C} 1 \mathrm{~s}$ spectra as a function of graphitic and pyridinic- $\mathrm{N}$ coverage during exposure to $\mathrm{N}$ plasma and after annealing. (filled circles) experimental results; (open circles) $\mathrm{C} 1 \mathrm{~s}$ shift calculated as explained in the text. The inset shows how the coverage of pyridinic (N2 + N3), pyrrolic (N4) and graphitic-N (N5 + N6) evolve upon annealing to 1040 and $1400 \mathrm{~K}$. desorption backshift of $\sim 180 \mathrm{meV}$. This behavior is consistent with an 341 $n$-type doping induced by the incorporation of graphitic nitrogen in 342 the carbon network which shifts the Dirac cone [44] as well as the 343 core levels towards higher binding energy. In a recent investigation of 344 $\mathrm{GR} / \mathrm{Ir}(111)$ [32], we observed a shift towards lower binding energy of 345 the $\mathrm{C} 1 \mathrm{~s}$ peak of graphene upon oxygen intercalation, accompanied by 346 a comparable shift of the Dirac point above the Fermi level, due to 347 hole-doping effects caused by charge transfer from carbon to the 348 oxygen covered Ir substrate. A similar argument can be used to interpret 349 the shift of the main graphene peak reported in Fig. 4, the only differ- 350 ence being the sign of the shift due to the $n$-type doping of graphitic 351 nitrogen. It is important to stress here that not only the nitrogen con- 352 centration but also its configuration affects the position of the Dirac 353 point. Previous investigations on graphene as well as carbon nanotubes 354 have shown that while graphitic nitrogen induces an $n$-type doping, the 355 pyridinic, pyrrolic and adatom or interstitial configurations cause a 356 p-type doping [25,45-47]. The largest effect is given by pyridinic nitro- 357 gen which, however, shifts the Dirac cone to lower binding energy by 358 about a third of the energy shift of graphitic-N [25]. This explains the ad- 359 ditional positive shift of the $\mathrm{C} 1 \mathrm{~s}$ observed upon the first annealing to 360 $1040 \mathrm{~K}$. Indeed, this determines an increase of the graphitic-N that 361 gives an $n$-type doping and, at the same time, a reduction of the 362 pyridinic/pyrrolic components leading to a $p$-type doping. The subse- 363 quent annealing to $1400 \mathrm{~K}$ results in a large, general loss of the nitrogen 364 atoms and, thus, the graphene peak moves back towards the undoped 365 energy position. This picture is further confirmed by a back-of-the- 366 envelope calculation of the $\mathrm{C}$ 1s shift as a function of the graphitic and 367 pyridinic coverage illustrated by the black open circles in Fig. 4 . The 368 shift was calculated using a contribution of +80 and $-25 \mathrm{meV} / \% \mathrm{ML} 369$ of graphitic and pyridinic nitrogen, respectively, assuming that only 370
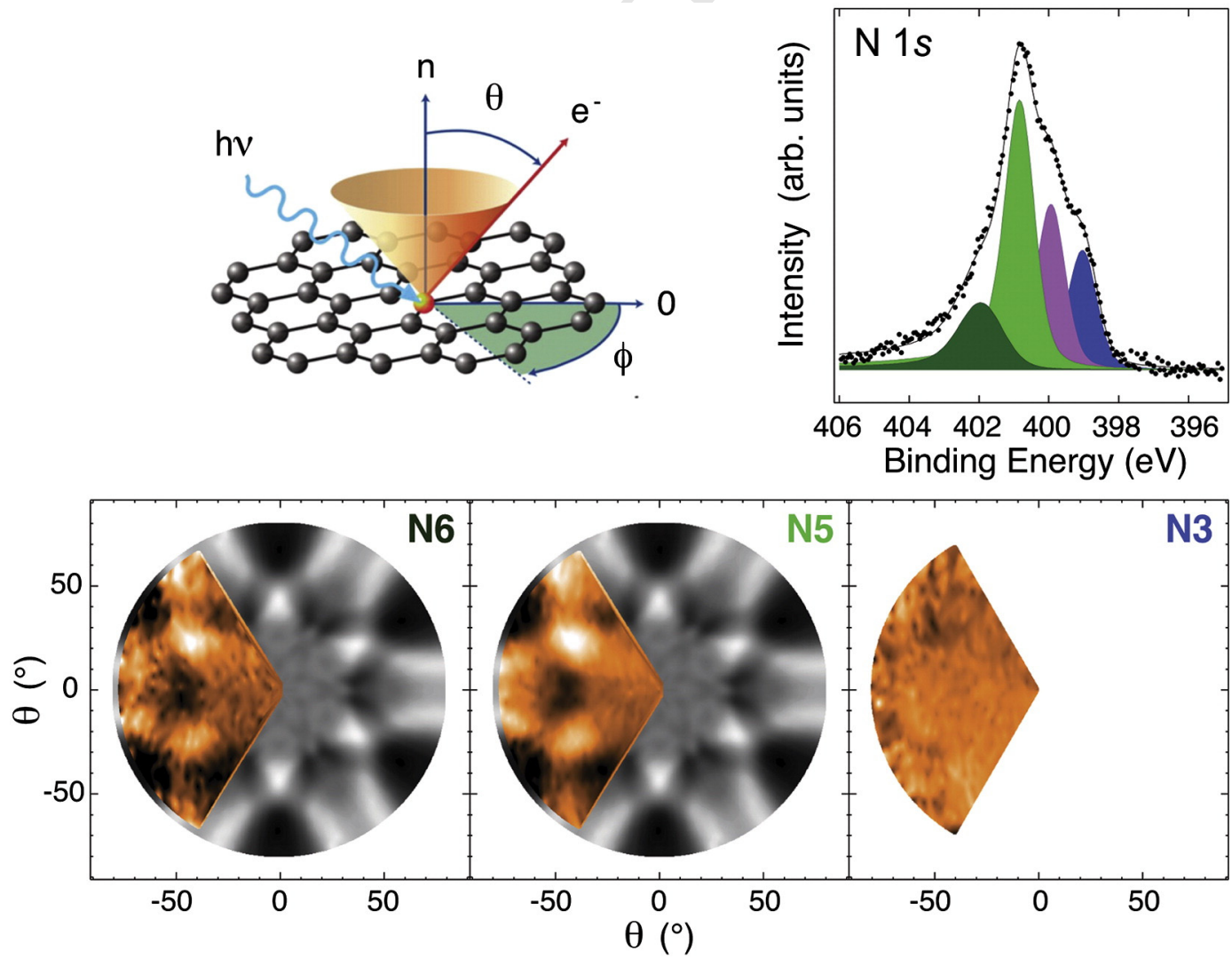

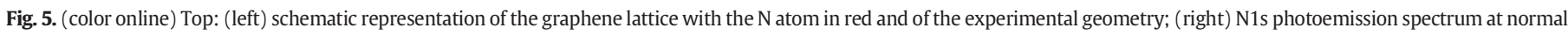

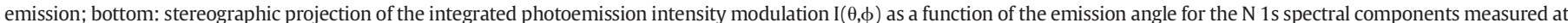

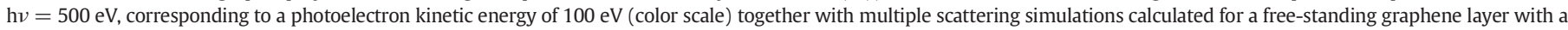

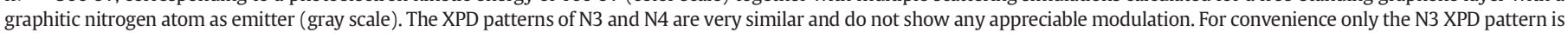
displayed on the right. (For interpretation of the references to color in this figure legend, the reader is referred to the web version of this article.) 
these nitrogen species contribute to the doping of the graphene layer (then excluding the pyrrolic-N contribution, which was found to be negligible [48], that of adsorbed and interstitial $\mathrm{N}$, which coverage is expected to be small, and the contribution of the nitrogen atoms adsorbed on the Ir surface). The good agreement between the experimental $C 1 \mathrm{~s}$ shift and the calculated one in the different situations further confirms the validity of this assumption. The graphitic-to-pyridinic contribution ratio to the $C 1 \mathrm{~s}$ shift of -3.2 of our work is in perfect agreement with that found in the same coverage range by Koch et al. [25], who calculated the shift of the Dirac point for the graphitic and pyridinic-N species for the $\mathrm{GR} / \mathrm{Au} / \mathrm{Ni}(111)$ system. This result indicates that the graphitic $n$-doping contribution is more than three times larger than the $p$-doping of the pyridinic species.

To gain further insight into the local environment of the $\mathrm{N}$ atoms, we performed XPD measurements by collecting $\mathrm{N} 1 \mathrm{~s}$ photoemission spectra at fixed photon energy of $500 \mathrm{eV}$ and different emission angles, similarly to the experiments reported in Refs. [49,50]. The measurements were carried out on a graphene layer exposed to $\mathrm{N}_{2}$ plasma for $15 \mathrm{~min}$, and subsequently annealed to $900 \mathrm{~K}$ in order to allow the conversion of the nitrogen species, with the maximum concentration of graphitic-N (see Fig. 3). However, it should be noted that in the present case the relative coverage of the $\mathrm{N} 3$ component due to pyridinic- $\mathrm{N}$ (Fig. 5) is higher than that of the sample discussed so far. This is most probably due to the more prolonged $\mathrm{N}$ plasma dose (15 min instead of $10 \mathrm{~min}$ ) which allows the formation of more pyridinic- $\mathrm{N}$, as can be extrapolated from Fig. 1d. The presence of all these components allowed us to get insight into the structure of graphitic, pyrrolic and pyridinic$\mathrm{N}$ species. The bottom part of Fig. 5 shows the N 1s XPD patterns of the N3-N6 components. In color scale are shown the experimental results while in gray scale is displayed the multiple scattering simulation calculated for a free-standing graphene layer with a graphitic nitrogen atom as the emitter. The striking similarity between the simulation and the measured diffraction patterns of N5 and N6 further confirms the assignment of these components to nitrogen in substitutional configuration. Moreover, these results confirm that the inclusion of graphitic nitrogen does not affect the local structural geometry of the carbon network. As to pyridinic and pyrrolic-N species, the absence of any measurable modulation in their XPD pattern shows that the configuration of these species does not preserve a locally ordered structure.

\section{Conclusions}

In this paper we report on the functionalization of a graphene layer grown on the $\operatorname{Ir}(111)$ surface by nitrogen plasma treatment. Different types of bonding configurations, namely, graphitic, pyridinic and pyrrolic nitrogen were identified by means of XPS and XPD techniques. Our results indicate that the GR-Ir interaction modulates the relative concentration of these nitrogen species, as it promotes the formation of pyrrolic- and pyridinic-N. Decoupling of GR from its Ir substrate by oxygen intercalation results in the depletion of the pyrrolic and pyridinic content accompanied by the enhancement of the substitutional component. The increase of the graphitic nitrogen concentration is obtained also by annealing the sample because of the higher thermal stability of this atomic configuration and of the selective conversion of the other nitrogen species. When the substitutional nitrogen is the most abundant configuration, a weak interaction between the Ir substrate and the nitrogen-doped graphene layer is re-established, which is similar to the undoped GR/Ir(111) interface. Finally, the XPD measurements gave a direct insight on the local geometry of the graphitic nitrogen atoms embedded in the carbon network. Graphitic-N was found to lie in the graphene plane while pyridinic and pyrrolic-N species do not show any modulation due to the lack of local order. The possibility to vary the concentration of nitrogen species by thermal annealing and by tuning the GR-substrate interaction is of importance for tailoring the electronic properties of monolayer graphene.

\section{Acknowledgments}

We acknowledge the financial support from MIUR through the pro- 435 ject PRIN entitled "GRAF. Frontiers in graphene research: understanding 436 and controlling advanced functionalities" (N.20105ZZYSE001) and from 437 the University of Trieste through the programme "Finanziamento di 438 Ateneo per progetti di ricerca scientifica - FRA 2014".

\section{References}

[1] F. Schwierz, Nat. Nanotechnol. 5 (2010) 487

[2] D. Zhan, J. Yan, L. Lai, Z. Ni, L. Liu, Z. Shen, Adv. Mater. 24 (2012) 4055

[3] W. Zhu, D. Neumayer, V. Perebeinos, P. Avouris, Nano Lett. 10 (2010) 3572.

[4] T. Ohta, A. Bostwick, T. Seyller, K. Horn, E. Rotenberg, Science 313 (2006) 951 Laegsgaard, A. Baraldi, S. Lizzit, Z Sljivancanin, F. Besenbacher, B. Hammer, T.G. 446 Pedersen, P. Hofmann, L. Hornekaer, Nat. Mater. 9 (2010) 315.

[6] F. Schedin, A.K. Geim, S.V. Morozov, E.W. Hill, P. Blake, M.I. Katsnelson, K.S. 448 Novoselov, Nat. Mater. 6 (2007) 652.

[7] F. Banhart, J. Kotakoski, A.V. Krasheninnikov, ACS Nano 5 (2011) 26 Balicas, F. Liu, P.M. Ajayan, Nat. Mater. 9 (2010) 430

Z-H. Sheng L. Shao, J-J Chen, W.-J. Bao, F.-B. Wang X.-H. Xia, ACS Nano 5 (2011) 453 4350.

[10] Y. Wang, Y. Shao, D.W. Matson, J. Li, Y. Lin, ACS Nano 4 (2010) 1790.

[11] D. Wei, Y. Liu, Y. Wang, H. Zhang, L. Huang, G. Yu, Nano Lett. 9 (2009) 1752.

[12] N. Li, Z. Wang, K. Zhao, Z. Shi, Z. Gu, S. Xu, Carbon 48 (2010) 255.

13] X. Li, W. Cai, J. An, S. Kim, J. Nah, D. Yang, R. Piner, A. Velamakanni, I. Jung, E. Tutuc, 458 S.K. Banerjee, L. Colombo, R.S. Ruoff, Science 324 (2009) 1312.

[14] Y.-C. Lin, C.-Y. Lin, P.-W. Chiu, Appl. Phys. Lett. 96 (2010) 133110.

15] M. Scardamaglia, B. Aleman, M. Amati, C. Ewels, P. Pochet, N. Reckinger, J.F. Colomer, 461 T. Skaltsas, N. Tagmatarchis, R. Snyders, L. Gregoratti, C. Bittencourt, Carbon 73462 (2014) 371.

[16] L. Zhao, R. He, K.T. Rim, T. Schiros, K.S. Kim, H. Zhou, C. Gutiérrez, S.P. Chockalingam, 464 C.J. Arguello, L. Pálová, D. Nordlund, M.S. Hybertsen, D.R. Reichman, T.F. Heinz, P. 465 Kim, A. Pinczuk, G.W. Flynn, A.N. Pasupathy, Science 333 (2011) 999.

[17] F. Joucken, Y. Tison, J. Lagoute, J. Dumont, D. Cabosart, B. Zheng, V. Repain, C. Chacon, 467 Y. Girard, A.R. Botello-Méndez, S. Rousset, R. Sporken, J.-C. Charlier, L. Henrard, Phys. 468 Rev. B 85 (2012) 161408.

[18] J.C. Meyer S. Kurasch, HJ. Park, V. Skakalova, D. Künzel, A Groß, A Chuvilin, G. 470 Algara-Siller, S. Roth, T. Iwasaki, U. Starke, J.H. Smet, U. Kaiser, Nat. Mater. 10471 (2011) 209.

19] C. Zhang, L. Fu, N. Liu, M. Liu, Y. Wang, Z. Liu, Adv. Mater. 23 (2011) 1020.

[20] S. Doniach, M. Sunjic, J. Phys. C 3 (1970) 285

[21] F.J. García de Abajo, M.A. Van Hove, C.S. Fadley, Phys. Rev. B 63 (2001) 075404.475

[22] K.-J. Kim, H. Lee, J. Choi, H. Lee, M.C. Jung, H.J. Shin, T.-H. Kang, B. Kim, S. Kim, J. Phys. 476 Condens. Matter 22 (2010) 045005.

[23] D. Usachov, O. Vilkov, A. Grüneis, D. Haberer, A. Fedorov, V.K. Adamchuk, A.B. 478 Preobrajenski, P. Dudin, A. Barinov, M. Oehzelt, C. Laubschat, D.V. Vyalikh, Nano 479 Lett. 11 (2011) 5401

[24] K.-j. Kim, S. Yang, Y. Park, M. Lee, B. Kim, H. Lee, J. Phys. Chem. C 117 (2013) 2129. 481

[25] R.J. Koch, M. Weser, W. Zhao, F. Viñes, K. Gotterbarm, S.M. Kozlov, O. Höfert, M. 482 Ostler, C. Papp, J. Gebhardt, H.P. Steinrück, A. Görling, T. Seyller, Phys. Rev. B 86483 (2012) 075401

[26] A. Mueller, M.G. Schwab, N. Encinas, D. Vollmer, H. Sachdev, K. Müllen, Carbon 84485 (2015) 426.

[27] R. Balog, M. Andersen, B. Jørgensen, Z. Sljivancanin, B. Hammer, A. Baraldi, R. 487 Larciprete, P. Hofmann, L. Hornekær, S. Lizzit, ACS Nano 7 (2013) 3823.

[28] P. Lacovig, M. Pozzo, D. Alfè, P. Vilmercati, A. Baraldi, S. Lizzit, Phys. Rev. Lett. 103489 (2009) 166101.

29] F. Presel, N. Jabeen, M. Pozzo, D. Curcio, L Omiciuolo, P. Lacovig S. Lizzit, D. Alfè, A. 491 Baraldi, Carbon 93 (2015) 187.

31] C.J. Weststrate, J.W. Bakker, A.C. Gluhoi, W. Ludwig B.E. Nieuwenhuys, Catal Today 494 154 (2010) 46.

[32] R. Larciprete, S. Ulstrup, P. Lacovig, M. Dalmiglio, M. Bianchi, F. Mazzola, L. 496 Hornekær, F. Orlando, A. Baraldi, P. Hofmann, S. Lizzit, ACS Nano 6 (2012) 9551. 497

[33] A. Barinov, O.B. Malcioğlu, S. Fabris, T. Sun, L. Gregoratti, M. Dalmiglio, M. Kiskinova, 498 J. Phys. Chem. C 113 (2009) 9009.

[34] L.H. Chan, K.H. Hong, D.Q. Xiao, T.C. Lin, S.H. Lai, W.J. Hsieh, H.C. Shih, Phys. Rev. B 70500 (2004) 125408

[35] M. Bianchi, D. Cassese, A. Cavallin, R. Comin, F. Orlando, L. Postregna, E. Golfetto, S. 502 Lizzit, A. Baraldi, New J. Phys. 11 (2009) 063002.

[36] R. Larciprete, S. Fabris, T. Sun, P. Lacovig, A. Baraldi, S. Lizzit, J. Am. Chem. Soc. 133504 (2011) 17315.

[37] A. Cavallin, M. Pozzo, C. Africh, A. Baraldi, E. Vesselli, C. Dri, G. Comelli, R. Larciprete, 506 P. Lacovig, S. Lizzit, D. Alfè, ACS Nano 6 (2012) 3034.

[38] C. Vo-Van, S. Schumacher, J. Coraux, V. Sessi, O. Fruchart, N.B. Brookes, P. Ohresser, T. 508 Michely, Appl. Phys. Lett. 99 (2011) 142504.

[39] S. Lizzit, R. Larciprete, P. Lacovig, K.L. Kostov, D. Menzel, ACS Nano 7 (2013) 4359. 510

[40] A. Baraldi, G. Comelli, S. Lizzit, D. Cocco, G. Paolucci, R. Rosei, Surf. Sci. 367 (1996) 511 L67. 
[41] A. Kumar, A. Ganguly, P. Papakonstantinou, J. Phys. Condens. Matter 24 (2012) 235503.

[42] F. Xu, M. Minniti, P. Barone, A. Sindona, A. Bonanno, A. Oliva, Carbon 46 (2008) 1489.

[43] E. Miniussi, M. Pozzo, A. Baraldi, E. Vesselli, R.R. Zhan, G. Comelli, T.O. Menteş, M.A. Niño, A. Locatelli, S. Lizzit, D. Alfè, Phys. Rev. Lett. 106 (2011) 216101.

[44] A. Lherbier, X. Blase, Y.-M. Niquet, F. Triozon, S. Roche, Phys. Rev. Lett. 101 (2008) 036808.

[45] M. Zhao, Y. Xia, J.P. Lewis, R. Zhang, J. Appl. Phys. 94 (2003) 2398.
46] S.H. Lim, R. Li, W. Ji, J. Lin, Phys. Rev. B 76 (2007) 195406.

[47] S. Jalili, R. Vaziri, Mol. Phys. 109 (2011) 687.

[48] B. Wang, L. Tsetseris, S.T. Pantelides, J. Mater. Chem. A 1 (2013) 14927. . Omiciuolo, N.G. Apostol, R. Larciprete, A. Baraldi, S. Lizzit, 524 ACS Nano 8 (2014) 12063.

[50] S. Lizzit, G. Zampieri, L. Petaccia, R. Larciprete, P. Lacovig, E.D.L. Rienks, G. Bihlmayer, 526 A. Baraldi, P. Hofmann, Nat. Phys. 6 (2010) 345. 